\title{
Summary and Concluding Remarks
}

\author{
Jean-Paul Zahn \\ LUTH, Observatoire de Paris, F-92195 Meudon, France
}

The last meeting on this subject, IAU colloquium 4, was held in 1969 at the Ohio State University in Columbus; it was organized by Arne Slettebak. Since then, much work has been accomplished in this field, but somehow no need was felt to hold another meeting, until this initiative taken by André Maeder and Philippe Eenens. They deserve our warm thanks for organizing this so successful venue, both socially and scientifically. I wish also to express our gratitude to the other members of the scientific organizing committee, and to those of the local organizing committee, in particular again to Philippe Eenens, to Ayda and Philip Jr., to Gloria Koenigsberger and to Luis Corral.

I found it extremely instructive to read the proceedings of the 1969 colloquium - there is no better way to measure the progress which has been made since, and that is why I will often refer to them in my brief summary. Among us there are three eye-witnesses of this progress, namely Helmut Abt, George Collins and Ian Roxburgh: not only did they attend the previous meeting, but also they there gave most interesting review talks.

\section{The Surface Rotation - through the Classical Method}

How much has the determination of the rotation speed through the classical spectroscopic method improved? This question was addressed by G. Collins in his comprehensive and lucid progress report. For moderate rotation, the accuracy of determining $v \sin i$ has increased enormously, but we have become more aware that the precision degrades as one approaches critical rotation, where the centrifugal force balances gravity. The reason is given by the famous Von Zeipel theorem, which states that at the surface of a rotating star, the radiation flux is proportional to the effective gravity, from which it follows that this flux can be much lower at the equator than on the poles. Therefore the regions where the rotation velocity is the largest contribute the least to the spectral line profile, which leads to a systematic underestimate of $v \sin i$ in the fastest rotators. This point was stressed also by I. Howarth and in a poster by Y. Frémat and collaborators.

D. Dravins explained us why we may hope to measure absolute wavelength shifts of late-type stars whose radial velocity has been determined accurately from astrometry. Since the effective gravity decreases with rotation speed, con- 
vection becomes more vigorous, and this produces a blueshift of spectral lines since the rising convective elements are brighter than the descending ones. Thus it seems possible to deduce the rotation speed from the intrinsic wavelength shift, and this method is being tested on the Hyades.

By treating high resolution spectra with Fourier transforms, A. Reiners and J. Schmitt were able to determine the amount of differential rotation in late-type stars. However this method can only be applied to spotless stars, because it requires symmetrical line profiles.

A large number of contributions reported the results of precise measurements of $v \sin i$, with the caveat mentioned above. Their enumeration demonstrates how actively observations are carried out, by numerous teams. D. Gies, P. North et al., W. Huang et al. and L. Corral et al. focused on OB stars in clusters and associations, whereas H. Levato \& M. Grosso dealt with southern B stars. The situation with Be stars was reviewed by J. Zorec, together with posters by S. Jankov et al. and by Levenhagen et al. Other contributions concerned later type stars (F. Fekel, F. Royer et al., J. R. Stauffer), red giants, HB and AGB stars (J. de Medeiros), horizontal branch stars (A. Recio-Blanco et al.) and metal poor evolved stars (J. Da Silva et al.). Of particular interest is the measurement of $v \sin i$ of $\mathrm{M}$ and $\mathrm{L}$ dwarfs, performed in the infrared by K. Hinkle et al. New results on pre-white dwarfs and on magnetic white dwarfs were presented respectively by T. Rauch et al., and by S. Jordan \& S. Friederich. Finally Philippe Eenens convinced us that it is a real challenge to determine the rotation speed of $\mathrm{B}[\mathrm{e}], \mathrm{LBV}$ and WR stars.

\section{The Surface Rotation - New Approaches}

New powerful techniques have been developed since 1969 to determine the surface rotation. Two of them make use of the inhomogeneities on the stellar surface: 'We love spots!' declared one of the speakers.

One is to analyze the photometric variations of spotted stars, and this has been applied with much success to PMS and T Tauri stars, as we heard from R. Mathieu and saw in the posters by M. Lamm et al. and N. Huélamo et al. Several groups work very actively with that method, and they have obtained a wealth of results, which show in particular that these stars must be coupled with a disk which brakes them down; otherwise they would be spinning much faster.

Another powerful technique is to measure the rotation of spotted stars through Doppler imaging, as was described by P. Petit and J.-F. Donati. By analyzing a number of stars, their team was able to show that the absolute differential rotation, i.e. the difference in angular velocity between equator and poles, does not vary much with the rotation speed, and that is always positive. In other words, fast rotators rotate more uniformly than slow rotators, such as the Sun.

And now interferometry is being applied to measure the angular size, and thus the shape of stars, which permits to deduce the rotation rate from the oblateness. G. van Belle reported the first results obtained on a main sequence star, namely Altair, for which a $v \sin i$ of $210 \mathrm{~km} / \mathrm{s}$ has been derived. Several teams are preparing observation campaigns at the VLTI, as was announced by 
Domiciano de Souza et al.: not only will the oblateness be determined, but stellar spots and large scale motions could also be detected through their chromatic signature.

\section{Rotation and Metallicity}

It is quite plausible that the angular momentum gathered during star formation is a function of metallicity. To verify whether this is true, a large survey have been undertaken by $\mathrm{F}$. Royer and colleagues to compare the $v \sin i$ distributions of B main sequence stars in clusters of different metallicity. The available data were reviewed and discussed by D. Gies.

A poster by R. Levenhagen and colleagues presents the results of a study which looked for variations of the rotational velocity of Be stars as a function of their location in the Galaxy. Another by J. Da Silva and colleagues focused on the rotation of metal-poor evolved stars.

No definite conclusion seems to have been drawn yet from these surveys.

\section{Rotation and Activity}

It has been known for a while already that magnetic activity is related to rotation. New results obtained through Doppler-Zeeman imaging were presented by J.-F. Donati, and they give now access to the magnetic topologies causing this activity. The data are of such quality that it becomes possible to investigate the feedback of the magnetic field on the (differential) rotation.

In brown dwarfs, fast rotation does not lead to enhanced activity, as was explained by G. Basri who reviewed the properties of these stars; thus the dynamo mechanism operating in them must differ from that in their stellar cousins, as he names them.

Finally T. Ayres gave a very interesting talk, describing how a star can get rid of a fossil field surviving from the PMS phase, thus opening the road for a cyclic dynamo, and for a coronal wind.

\section{Interior Rotation}

The 1969 colloquium was held in the midst of a heated debate about the internal rotation of the Sun. R. Dicke had been claiming for some time that the solar core was spinning much more rapidly than the surface, and there he presented the instrument he had built to measure the oblateness of the Sun. His opponents, namely L. Howard, D. Moore and E. Spiegel, had earlier replied that such a fast spinning core was not possible because during the spin-down of the Sun through its magnetized wind, the surface rotation would spread into the interior due to radiative diffusion. Both sides were wrong. Thanks to helioseismology, we have learned that the radiative interior is in quasi uniform rotation, with an angular velocity which is the average of that observed on the surface. But, as we shall see below, this uniform rotation cannot be achieved through a radiation driven circulation alone, as suggested by Howard et al.; another physical process must be invoked for that. 
At our symposium, it was J. Christensen-Dalsgaard who presented the beautiful achievements of helioseismology, in particular concerning the internal rotation. There is still some uncertainty about the rotation of the innermost core of the Sun, which could even be spinning slower than the surface, as was discussed by $\mathrm{S}$. Turck-Chièze.

The same technique is being used to probe the interior rotation of pulsating stars, although with much less observed frequencies than in the Sun, which renders the mode identification more difficult. The situation of the $\delta$ Scuti stars was reviewed by J. Matthews, and that of massive stars by C. Aerts. The effect of rotation on stellar oscillations was illustrated by R. Townsend in colorful animations, and a poster by $\mathrm{F}$. Lignières and M. Rieutord presented new results on the oscillations of rotating, hence flattened, polytropes, using a non-perturbative approach.

Soon we will get observations of the oscillations of late-type stars, which are generated through stochastic excitation by the convective motions, as in the Sun. I. Roxburgh reviewed the planned space missions in Europe: MONS (Denmark), COROT (France) and Eddington, built by ESA and to be launched by 2008. J. Matthews gave a presentation of MOST, the Canadian instrument, which should be launched in April 2003.

\section{Be Stars: the Fastest Rotators}

In 1969 , the Be stars were already recognized as the fastest rotators, but no mention was made there about the existence of a disk: J. Hutchings, for instance, spoke about an 'extended atmosphere', while D. Limber presented a curious mechanical toy model with springs and spokes to explain the observed temporal variations. Much progress has been achieved since, which has been reviewed by J. Zorec, but there is still debate on whether most B stars undergo a Be phase, and where in the HR diagram the phenomenon appears. In several posters with his collaborators, he presented new observational results, and addressed other problems concerning these stars, such as their initial mass function.

S. Owocki described very convincingly how matter can be 'launched' like a rocket from a star spinning near critical rotation, and thus form a disk; he insisted that 'radiation is enemy of disks'. A. Okazaki also discussed the formation and dissipation of viscous disks, whereas M. Maintz and collaborators, in their poster, reported on their search for proofs of the spin-up scenario.

\section{Tidal Interaction in Close Binaries}

Through tidal interaction, angular momentum is exchanged between the components of binary stars and their orbit, while kinetic energy is transformed into heat. When the stars are close enough, this results in the synchronization of their rotation with the orbital motion, and in the circularization of their orbits. These general properties were recalled by G. Koenigsberger; new observational results were presented by S. Meibom on cluster stars, and by H. Abt, in his poster, on field stars.

Two physical mechanisms have been identified, which are responsible for the tidal dissipation and hence for the dynamical evolution of close binary stars: 
turbulent dissipation in stars which possess an outer convection zone and radiative damping in stars with an outer radiation zone. These processes were discussed by M. Rieutord, who also presented another mechanism, invoking the elliptic instability observed in the laboratory, to account for the circularization of the oldest binaries, which seems to proceed faster than predicted by the two mechanisms mentioned above.

\section{Abundance Anomalies in Rotating Stars}

In standard models of stellar structure, radiation zones are treated as an inert medium, with no other motions than rotation. But there are many signs that some mixing must occur within these zones. On the surface of somewhat evolved massive main sequence stars, chemical elements are detected which can only be synthesized in the inner core. This was documented by several contributions: the talk by A. Herrero and his co-worker D.J. Lennon on main sequence OB stars, the poster by P. Crowther and Ch. Evans on OB stars in the Magellanic Clouds, confirmed by the poster by S. Heap and Th. Lanz on the SMC. Y. Frémat and collaborators concentrated on the light element abundances in a Be star seen pole-on.

In intermediate and low mass stars, it is the lithium depletion which reveals that some mixing must occur below the convection zone, beyond reasonable overshoot. The relation between $\mathrm{Li}$ abundance and rotation in subgiants and giants was discussed by B. Barbuy and S. Mallik, and also in several posters by N. Drake et al., R. Konstantinova-Antova, Lèbre et al., and Do Nascimento Jr. et al. The results of abundance measurements of CNO and other heavy elements in RS CVn stars were presented in a poster by S. Beryugina and her collaborators.

The status of Ap, Am and HgMn stars was reviewed by G. Mathys. Such stars are well known to present pronounced abundance anomalies, because microscopic processes such as diffusion, radiative levitation and gravitational settling can proceed without much interference by turbulent motions.

A very important contribution came from S. Balachandran, who demonstrated that the Li depletion is not simply linked with the loss of angular momentum, as it is predicted by some theories. She re-analyzed the spectra of two tidally-locked binaries in the Hyades, for which it had been claimed earlier that they had preserved some of their lithium. She showed convincingly that this was not the case, which puts a strong constraint on the angular momentum transport in these stars.

\section{Modeling Rotating Stars}

The main mechanisms which produce mixing in a stellar radiation zone are the thermally driven meridional circulation plus, most likely, turbulence. This was recognized already in 1969, based on earlier work by Eddington, Vogt, Sweet and Mestel. I. Roxburgh summarized it then in one sentence: "Inside a rapidly rotating star we expect a battle between the meridional circulation and the turbulence driven by the differential rotation." 
At the present symposium, these processes were reviewed by S. Talon, and in her talk P. Garaud focused on properties of the meridional circulation. The modelization of shear turbulence was further addressed in posters by $F$. Lignières and by $R$. Hirschi and his Geneva team. As explained by S. Talon, and illustrated later by A. Maeder, rotational mixing with meridional circulation and turbulence accounts rather well for the properties of rapid rotators, the main uncertainty being the recipes used to model the turbulent transport. In slow rotators, however, these processes are much less efficient, and the fact that the solar interior is rotating almost uniformly proves that another mechanism must be operating there. This point was also stressed by S. Balachandran and M. Pinsonneault.

One of these mechanisms may be magnetic stresses, due for instance to a fossil field. The role of magnetic fields was reviewed by $\mathrm{P}$. Charbonneau, who threatened jokingly that "if you ignore magnetic fields, you will be sorry!" His opinion was shared by I. Roxburgh, when he chaired one of the general discussions. H. Spruit, on the other hand, developed arguments in favor of a dynamo operating in the radiation zone.

Another possibility is the transport of angular momentum through long period gravity waves which are emitted at the base of the convection zone. This process was presented by S. Talon, who has performed numerical simulations showing that such waves could extract angular momentum quite efficiently, perhaps even producing a slowly rotating core.

Observational evidence is needed to choose between these two mechanisms, and to assess the role and the strength of turbulence in radiation zones. An extremely precious indicator is provided by the abundance anomalies at the surface of some stars, in particular the A-type stars. In a quiet medium, chemical elements undergo gravitational settling or radiative levitation, and this is observed to some extent. However the abundance anomalies would be much stronger than observed if these microscopic processes were not inhibited to a certain degree by large scale advection or turbulent diffusion. Thus the departure from 'normal' abundances puts a constraint on the mixing processes, as was demonstrated by G. Michaud.

In 1969 , no one doubted that the rotation of the solar convection zone should comply with the Taylor-Proudman theorem, namely that the angular velocity should be constant on cylinders. This was illustrated by the model presented by $M$. Clement, for instance. But that picture has been shattered by the advent of helioseismology, which permitted to discover that the angular velocity barely changes with depth in the convection zone. Since, it has been a challenge to reproduce this property in numerical simulations. J. Toomre reported that he and $\mathrm{S}$. Brun recently succeeded in obtaining a rotation profile which resembles that of the Sun, by increasing the spatial resolution, and thus allowing for more 'turbulent' motions. Their 3D code has been adapted to treat likewise a convective core, and S. Brun presented the first results of the Boulder team on modeling the deep interior of an A-type star; a poster of M. Browning et al. gives more details on the simulation, and shows the behavior of magnetic field in such a star. R. Deupree displayed the results of his $2 \mathrm{D}$ simulations. 


\section{The Evolution of Rotating Stars}

During the short pre-main sequence evolution, it is not necessary to worry about the transport of angular momentum: it suffices to account for its conservation as the star contracts non-homologously. Detailed calculations of PMS evolution were presented by S. Sofia and S. Barnes, and by S. Wolff and her collaborators. The main uncertainty resides in the initial conditions, namely when and how the star decouples from its circumstellar disk, which probably depends on the strength of the magnetic field.

On the main sequence and in later evolutionary phases, mixing in the rotation zones plays an increasing role, and to model this most calculations invoke the rotational mixing described above, based on meridional circulation and on shear turbulence. There are some differences in the recipes for the turbulent transport, and not everyone takes into account the distinct advective character of the angular momentum transport by the meridional circulation. To illustrate what the difference is between advection and diffusion, André Maeder has this convincing argument: "If the circulation of money were a diffusive process, money would go from the pocket of the rich to that of the poor. Since that is not observed, it must be an advective process." And true enough, models with the correct advective transport show that angular momentum is transported up the gradient of angular velocity.

The most extensive calculations are those performed by the Geneva team on massive stars, and their results were presented by A. Maeder. Not only do they explain the abundance anomalies detected on the surface of these stars (enrichment in ${ }^{4} \mathrm{He}$ and ${ }^{14} \mathrm{~N}$ ), but also the observed ratio between blue and red supergiants. At lower metallicity, the angular velocity gradient is steeper, producing stronger mixing. Mass loss is included, and particular care is taken to model the wind, which can be stronger at the equator than at the poles, or the opposite, depending on temperature, and this obviously has an impact on the efficiency of angular momentum loss. H. Lamers reviewed what the observations tell us about mass loss, whereas A. Nota and S. Kwok focused on the ejecta, with the latter attempting to interpret the various shapes of planetary nebulae.

M. Pinsonneault described the evolution of rotating solar-type stars, and he recalled that rotational mixing alone would leave a fast rotating core in the Sun. A poster by $\mathrm{S}$. Théado and $\mathrm{S}$. Vauclair illustrated the quenching of the meridional circulation by the built-up of molecular weight barriers, due to gravitational settling. S. Turck-Chièze demonstrated how mixing confined in the tachocline could explain the depletion of lithium, while preserving beryllium.

Several contributions dealt with the post main sequence evolution. C. Charbonnel showed that rotational mixing can explain the surface composition of subgiants, in particular the enrichment in ${ }^{13} \mathrm{C}$, whereas $\mathrm{F}$. Herwig described the impact of rotation on the s-process in AGB stars.

The effect of rotation on advanced phases was addressed by G. Meynet, who showed that it favors the formation of Wolf-Rayet stars. S. Kawaler discussed the rotation of white dwarfs, which results from the coupling of the cores of AGB stars with their envelopes. White dwarfs in binary systems were considered by S. Starrfield; known as cataclysmic variables, they accrete matter from their companion when it overflows its Roche lobe, thus causing the nova phenomenon. 
A collaborative work on pre-supernova evolution was described by A. Heger, with rotational mixing and including the effect of magnetic field; one goal was to explain the rotation of pulsars. Separately, N. Langer reported on the evolution of binary stars. S. Woosley closed the symposium with a brilliant overview of the supernova phenomenon, emphasizing its connection with the cosmic gamma-ray bursts.

\section{Conclusion}

I hope that the reader has not been bored by this lengthy enumeration, whose purpose was to stress that so much progress has been achieved. Major advances occurred during the last ten years, thanks to observational data of ever increasing accuracy and completeness, which put now much stringer constraints on theory. The modeling of rotating stars begins to spread, although it is still in a rather crude state. We know what is required to better describe the effects of rotation on stellar evolution: we need more reliable prescriptions for the turbulent transport, and we must come up with a self-consistent treatment of the magnetic transport.

We are all aware that a bright future is promised to stellar physics, and in particular for stellar rotation, thanks to three techniques which are improving at very fast pace: asteroseismology, soon carried out in space; interferometry, in infrared and visible; and numerical simulations of high spatial resolution.

Of course, many open questions remain, but I am confident that many of these will be answered until we reconvene at the next symposium on stellar rotation. I do hope that we will not wait another 33 years for meeting again, although we could then celebrate my 100th birthday! 